\title{
A Fully Automatic Multiscale 3-Dimensional Hessian-Based Algorithm for Vessel Detection in Breast DCE-MRI
}

\author{
Anna Vignati, PhD, * Valentina Giannini, PhD, * Alberto Bert, PhD, $\dagger$ Pasquale Borrelli, MS, $\neq$ \\ Massimo De Luca, PhD, * Laura Martincich, MD, * Francesco Sardanelli, MD, $\$ / /$ and Daniele Regge, MD*
}

\begin{abstract}
Objectives: The objectives of this study were to develop a fully automatic method for detecting blood vessels in dynamic contrast-enhanced magnetic resonance imaging of the breast on the basis of a multiscale 3-dimensional Hessian-based algorithm and to evaluate the improvement in reducing the number of vessel voxels incorrectly classified as parenchymal lesions by a computer-aided diagnosis (CAD) system.
\end{abstract}

Materials and Methods: The algorithm has been conceived to work on images obtained with different sequences, different acquisition parameters, such as the use of fat-saturation, and different contrast agents. The analysis was performed on 28 dynamic contrast-enhanced magnetic resonance imaging examinations, with 39 malignant ( 28 principal and 11 satellite) and 8 benign lesions, acquired at 2 centers using 2 different 1.5-T magnetic resonance scanners, radiofrequency coils, and contrast agents (14 studies from group A and 14 studies from group B). The method consists of 2 main steps: $(a)$ the detection of linear structures on 3-dimensional images, with a multiscale analysis based on the second-order image derivatives and $(b)$ the exclusion of non-vessel enhancements based on their morphological properties through the evaluation of the covariance matrix eigenvalues. To evaluate the algorithm performances, the identified vessels were converted into a 2-dimensional vasculature skeleton and then compared with manual tracking performed by an expert radiologist. When assessing the outcome of the algorithm performances in identifying vascular structures, the following terms must be considered: the correct-detection rate refers to pixels identified by both the algorithm and the radiologist, the missed-detection rate refers to pixels detected only by the radiologist, and the incorrect-detection rate refers to pixels detected only by the algorithm. The Wilcoxon rank sum test was used to assess differences between the performances of the 2 subgroups of images obtained from the different scanners.

Results: For the testing set, which is composed of 28 patients from 2 different clinical centers, the median correct-detection rate was $89.1 \%$, the median missed-detection rate was $10.9 \%$, and the median incorrect-detection rate was $27.1 \%$. The difference between group A and group B was not significant $(P>0.25)$. The exclusion of vascular voxels from the lesion detection map of a CAD system leads to a reduction of $68.4 \%$ (30.0\%) (mean [SD]) of the total number of false-positives because of vessels, without a significant difference between the 2 subgroups $(P=0.50)$.

Received for publication March 29, 2012; and accepted for publication (after revision) July 30, 2012.

From the *Department of Radiology, IRC@C: Institute for Cancer Research at Candiolo, Candiolo; †im3D S.p.A, Turin; $\$$ Radiologia e Medicina Nucleare, Dipartimento di Scienze Biomorfologiche Funzionali, Università degli Studi di Napoli Federico II, Napoli; §Dipartimento di Scienze Medico-Chirurgiche, Università degli Studi di Milano, Milan; and |IRCCS Policlinico San Donato, Milan, Italy.

Conflicts of interest and source of funding: F. Sardanelli received research grants from Bayer Pharma AG and from Bracco Imaging Group, and is on the speakers' bureau for Bayer Pharma AG. A. Bert is a researcher at im3D S.p.A. L. Martincich was a blinded reader for Bracco Imaging Group and Bayer Schering Pharma, was a speaker for Bracco Imaging Group, was a board member of GE Health care, and developed educational presentations for ABC Medical Imaging. D. Regge was a consultant for im3D S.p.A. and is currently paid by Springer Editor for manuscript preparation. For the remaining authors, none were declared.

Reprints: Anna Vignati, PhD, Department of Radiology, IRC@C: Institute for Cancer Research at Candiolo, Strada Provinciale $142 \mathrm{Km} 3.95$, 10060 Candiolo, Torino, Italy. E-mail: anna.vignati@ircc.it.

Copyright (C) 2012 by Lippincott Williams \& Wilkins

ISSN: 0020-9996/12/4712-0705
Conclusions: The system showed promising results in detecting most vessels identified by an expert radiologist on both fat-saturated and non-fat-saturated images obtained from different scanners with variable temporal and spatial resolutions and types of contrast agent. Moreover, the algorithm may reduce the labeling of vascular voxels as parenchymal lesions by a CAD system for breast magnetic resonance imaging, improving the $\mathrm{CAD}$ specificity and, consequently, further stimulating the use of CAD systems in clinical workflow.

Key Words: DCE-MRI, computer-aided diagnosis, breast cancer, vessel-detection algorithm

(Invest Radiol 2012;47: 705-710)

B reast cancer is the second most common neoplasia after lung cancer and is the most common cancer in women. ${ }^{1-3}$ Dynamic contrast-enhanced magnetic resonance imaging (DCE-MRI) is a noninvasive imaging technique used more and more frequently in breast cancer diagnosis. ${ }^{4,5}$ A recent multicenter study showed that breast DCE-MRI used to screen women at high risk for breast cancer has $91 \%$ sensitivity, $97.4 \%$ specificity, and a $62 \%$ positive predictive value. ${ }^{6}$ However, with the increasing use of breast MRI, the large numbers of images that need to be interpreted render the accuracy and efficiency of interpretation an ever more challenging issue. ${ }^{7,8}$

Recent studies aimed to increase specificity of DCE-MRI by adding a breast vascularity analysis to the standard morphologic and kinetic criteria, demonstrating the association between the 1-sided increased vascularity and ipsilateral breast cancer. ${ }^{9-12}$ This type of analysis is part of recent investigations exploring the diagnostic value of vessel analysis. During the past decade, research has approached this issue in 2 ways: first, by investigating whole-breast vascularity and second, by examining local vascularity (ie, vessels adjacent to and feeding a lesion or lesions). ${ }^{13}$ From 2001 to 2005 , several studies $^{9-11,14}$ considered the association between asymmetric increase in whole-breast vascularity and ipsilateral cancer, reporting sensitivity values ranging from $66 \%$ to $88 \%$ and specificity values from $57 \%$ to $100 \%$. In 2008 , Schmitz et al ${ }^{12}$ increased specificity by $13 \%$ without affecting sensitivity after adjustment for vascularity of the breast to standard morphologic and kinetic analyses. In the matter of local vascularity, studies exploring the presence of vessels adjacent to a lesion or lesions reported sensitivity values ranging from $47 \%$ to $89 \%$ and specificity values ranging from $83 \%$ to $88 \%{ }^{14-16}$ All reported studies on vascularity findings, which are not usually evaluated by radiologists, suggested further investigation of vessel analysis on contrast-enhanced breast magnetic resonance images.

Most vessels can be identified on maximum intensity projections (MIPs); however, evaluating vessels using multiple views may be time consuming for radiologists. ${ }^{8}$ Therefore, the automatic detection and extraction of a breast vascular map may improve the workflow of radiologists and may have clinical relevance.

Several studies have tried to address this problem: Frangi et $\mathrm{al}^{17}$ presented a multiscale vessel enhancement filtering method based on Hessian matrix eigenvalues to detect linear structures, whereas Sato et $\mathrm{al}^{18}$ described a multiscale 3 -dimensional line filtering method 
based on directional second-order derivatives of Gaussian-smoothed images to highlight vessels in 3-dimensional images. Both previously mentioned algorithms were developed to extract vessels on images from magnetic resonance angiography, a well-established technique for imaging of the vascular system characterized by high signal-tonoise ratio. Applying those methods to DCE-MRI images is not suitable because MRI images are characterized by a noisy background, mainly because of the fibroglandular tissue. To the best of our knowledge, only 1 study $^{8}$ developed an algorithm to detect blood vessels in breast DCE-MRI, based on wavelet transform and the Hessian matrix. However, this method has several limitations: it has been tested only on non-fat-saturated images; it relies on a preliminary manual interaction while breast lesions are excluded by placing a square box over the lesion; and it has been tested on patients with relatively large lesions (mean [SD], 4.0 [2.1] cm), large vessels, and no motion artifacts on subtracted images.

The principal purpose of this study was to implement a fully automatic algorithm for vessel detection on breast DCE-MRI that is able to deal with images obtained with different sequences, different acquisition parameters, such as the use of fat-saturation (fat-sat) or non-fat-saturation, and different types of contrast agents. The method first extracts the linear structures from 3-dimensional images by means of a filter bank technique based on the Hessian matrix, then the non-vessel-detected voxels are discarded by using an image covariance matrix. At the end of the process, a 3-dimensional vasculature map is built.

The second goal of the current study was to demonstrate the ability of the algorithm to reduce the number of vessel false-positives (FPs) of a computer-aided diagnosis (CAD) system, commonly used to extract and display information from hundreds of images to help radiologists' reading.

\section{MATERIALS AND METHODS}

\section{Patients and Lesions}

A total of 28 sequential DCE-MR examinations from patients with ages ranging from 28 to 76 years (mean [SD], 46.8 [14.5] years), with 39 malignant ( 28 principal and 11 satellite) and 8 benign tumors, were used to test the system. The reference standard was given through histopathology using needle biopsy or by a follow-up for benign lesions and through histopathology using surgical specimen for malignant lesions. The lesion sizes ranged from 5 to $117 \mathrm{~mm}$ (mean [SD], 24.4 [20.5] mm). Studies were collected from 2 institutions, each of which used different MRI equipment and a different protocol. The Local Ethics Committee approved the retrospective use of the database for scientific purposes and waived off informed consent. The study was conducted in accordance with national legislation and the Declaration of Helsinki.

\section{MRI Protocols}

Group A included 14 studies acquired on a 1.5 -T scanner (Signa Excite HDx; General Electric Healthcare, Milwaukee, Illinois) using an 8-channel breast radiofrequency (RF) coil and a fat-sat 3-dimensional axial fast spoiled gradient-echo sequence (VIBRANT; General Electric Healthcare) with the following technical parameters: TR/TE, 4.5/2.2 millliseconds; flip angle, 15 degrees; reconstructed matrix, $512 \times 512$; field of view, $320 \mathrm{~mm}$; slice thickness, $2.6 \mathrm{~mm}$; and pixel size, $0.625 \times 0.625 \mathrm{~mm}^{2}$. A total of 7 scans were acquired for each study: 1 baseline, 5 contrast-enhanced frames with a time resolution of 50 seconds, and 1 delayed frame acquired 7 minutes after contrast injection. Gadopentetate dimeglumine (Magnevist; Bayer-Schering, Berlin, Germany) was administered at a dose of $0.1 \mathrm{mmol} / \mathrm{kg}$ at $2 \mathrm{~mL} / \mathrm{s}$, followed by $20 \mathrm{~mL}$ of saline solution at the same rate.

Group B comprised 14 studies performed on a different 1.5-T scanner (Sonata Maestro Class; Siemens, Erlangen, Germany) using a dynamic 3-dimensional axial spoiled fast low-angle shot sequence and a 4-element 2-channel coil, with the following technical parameters: TR/TE, 11/4.9 milliseconds; flip angle, 25 degrees; matrix, $512 \times 512$; field of view, $384 \mathrm{~mm}$; slice thickness, $1.3 \mathrm{~mm}$; and pixel size, $0.75 \times 0.75 \mathrm{~mm}^{2}$. Gadobenate dimeglumine (MultiHance; Bracco Imaging, Milan, Italy) was used as contrast agent, administered at a dose of $0.1 \mathrm{mmol} / \mathrm{kg}$ at $2 \mathrm{~mL} / \mathrm{s}$, followed by $20 \mathrm{~mL}$ of saline solution at the same rate. One baseline scan was acquired before contrast injection, followed by 5 contrast-enhanced frames taken $118 \mathrm{sec}-$ onds apart. Fat-sat sequences were not performed in group B patients.

\section{Image Processing}

The algorithm was conceived as a 2-step process to search for geometrical tubelike structures (Fig. 1). The first step, a multiscale analysis, evaluated second derivatives to extract linear structures (Fig. 1A), and the second step reduced non-vessel-detected voxels on the basis of the analysis of the covariance matrix (Fig. 1B). The isotropic image difference between the enhanced frame acquired approximately 2 minutes after injection and the unenhanced frame was considered for vessel analysis. In particular, the second enhanced image was considered for group A (acquired 100 seconds after contrast injection) and the first enhanced image was considered for group $\mathrm{B}$ (acquired 118 seconds after contrast injection). In this way, the best "angiographic effect" for both the arteries and the veins was obtained. In fact, in the subsequent acquisitions, a more pronounced

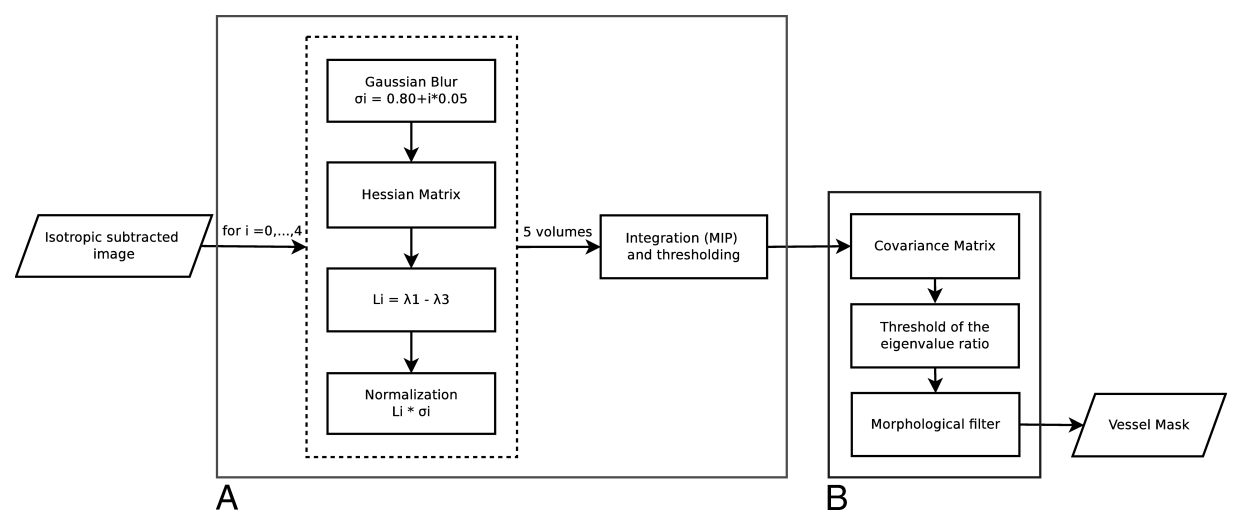

FIGURE 1. Algorithm pipeline. Box A represents the vessel-detection step, which is composed of the multiscale analysis (dot-line box) and successive volume integration, whereas box B is the non-vessel-detected voxel reduction step. 
distribution of the contrast agent in the interstitial space reduced vascular enhancement. ${ }^{11}$

\section{Multiscale Analysis}

To extract vessels of different dimensions, a measurement scale that varies within a set range was introduced by applying a Gaussian blur filter using 5 different SDs (sigma) so that 5 subimages smoothed with increasing sigma size were obtained. In fact, although vessels with a diameter greater than $2 \mathrm{~mm}$ are always extracted at every sigma, introducing multiresolution analysis makes it possible to extract vessels of diameters comprised between 1 and $2 \mathrm{~mm}$ as well. Sato et $\mathrm{al}^{18}$ reported that the sigma of Gaussian blur and vessel dimension are related according to the following formula:

$$
\sigma=\mathrm{r} \sqrt{2}
$$

where $r$ is the vessel radius.

The sigma values ranged from $0.80\left(\sigma_{1}\right)$ to $1.00\left(\sigma_{5}\right)$, within a step of 0.05 .

To enhance and extract vessels on each smoothed image, the morphology of curvilinear structures was assessed via eigenvalue analysis of the Hessian matrix. The Hessian matrix $H$ describes the second-order structures of local intensity variations on gray-scale images around each point of a 3-dimensional image, having the potential to highlight different components in an image. ${ }^{19}$

When the eigenvalues of $H$ are $\lambda_{1}, \lambda_{2}, \lambda_{3}\left(\lambda_{1}>\lambda_{2}>\lambda_{3}\right)$ and their corresponding eigenvectors are $e_{1}, e_{2}$, and $e_{3}$, respectively, the eigenvector $e_{1}$, corresponding to the largest eigenvalue $\lambda_{1}$, represents the direction along which the second derivative is the maximum, and $\lambda_{1}$ gives the maximum second derivative value (whereas $\lambda_{3}$ represents the minimum second derivative value). ${ }^{8,20}$

Both $\lambda_{2}$ and $\lambda_{3}$ have a minimum value at the center of a bright 3-dimensional line, whereas $\lambda_{1}$ approximates zero. Therefore, the conditions of a bright line can be regarded as the following:

$$
\begin{gathered}
\lambda_{1} \simeq 0 \\
\lambda_{2} \simeq \lambda_{3} \ll 0 .
\end{gathered}
$$

The eigenvalues of the Hessian matrix were evaluated after setting all positive eigenvalues to zero. By and large, the profiles of $\lambda_{1}$ and $\lambda_{3}$ are similar for the nodular structures, both showing negative values, whereas, for linear structures, $\lambda_{3}$ is negative and $\lambda_{1}$ tends to zero. Subtracting $\lambda_{3}$ from $\lambda_{1}$, the algorithm is able to distinguish linear structures having positive values from nodular structures having values that approximate zero. 8,20

To integrate information from the multiscale analysis and for a fair comparison of images at multiple scales, for each scale, the obtained subimage was multiplied by the respective sigma value and the MIP of the 5 subimages was performed.

To compensate for the dependence on the breast, vessels, and lesion extension of a cumulative histogram-based threshold, the obtained MIP was dichotomized according to a threshold automatically calculated from the mammary vessel region. A suitable region of interest of a fixed size $(50 \mathrm{~mm} \times 100 \mathrm{~mm})$ was automatically selected in the region of mammary vessels of the MIP according to the method of Vignati et $\mathrm{al}^{21}$ and $99 \%$ of the cumulative histogram of the region of interest was chosen as the threshold to extract vessellike structures.

\section{Reduction of the Number of Non-vessel-detected Voxels}

The binary mask obtained in the previous step still contained some non-vessel structures, such as noise components and irregular margins of lesions. These regions have a blob-like structure with an elongated shape, characterized by positive values when minimum Hessian eigenvalues are subtracted from maximum Hessian eigenvalues.
To discard these findings on the binary mask, a method based on the covariance matrix was applied. The covariance is a measure of the "spread" of a set of points around their center of mass (mean); thus, it measures how much each of the dimensions varies from the mean with respect to the others.

For each voxel defined as a vessel in the previous step, the covariance was evaluated in an $8-\mathrm{mm}$ radius volume of interest. The eigenvectors of the covariance matrix indicate the principal direction of the analyzed region, and the eigenvalues represent the variance of the voxel coordinates along each eigenvector. Therefore, the covariance matrix is used as a morphological descriptor of a region in terms of the extension along its principal component. In this analysis, only the maximum $\left(\lambda_{3}\right)$ and the minimum $\left(\lambda_{1}\right)$ eigenvalues of the covariance matrix were considered because they make it possible to distinguish between linear $\left(\lambda_{3}>>\lambda_{1}\right)$ and elongated blob-like $\left(\lambda_{3}>\lambda_{1}\right)$ structures. Objects with the maximum and minimum eigenvalue ratio greater than 7 were extracted. Finally, objects with a volume less than $10 \mathrm{~mm}^{3}$ were discarded to exclude small isolated structures not connected to other vessel voxels.

\section{Data Evaluation and Statistical Analysis}

The manual vessel tracking performed by a radiologist with 10 years of experience in interpreting breast MRI images represented the reference standard. On the basis of anatomic distribution, branching, and morphology, the radiologist subjectively evaluated whether a linear structure was a vessel and he was provided with a self-made plug-in of ImageJ (a public domain, Java-based image processing program) to draw vessels on the 2-dimensional axial MIP of the subtracted image. During manual segmentation, the lesions were masked out to allow segmentation of vessels otherwise hidden by the lesions on the MIP.

The radiologist produced 2 different masks: the first one (standard reference map) included vessels with an estimated diameter greater than $1 \mathrm{~mm}$ and the second one (control map) included all other visible vessels. The radiologist also outlined the profile of the rib cage, and the pixels outside the region of clinical interest were not considered for the evaluation of system performances. The manual tracking procedure took about 10 minutes per case.

To quantify the algorithm-detected vessels, masks generated by the system were skeletonized and compared with the vascular track outlined by the radiologist. Because the skeleton and the track drawn by the radiologist may be within the same vessel but at a slightly off-pixel location, a vascular pixel in the skeleton determined by the algorithm and a pixel in the radiologist's track within a 3-pixel difference were considered as matching pixels.

A pixel in the algorithm skeleton matching a standard reference map pixel was considered correctly detected. A pixel in the algorithm skeleton without a matching pixel either in the standard reference map or in the control map was considered as a non-vessel-detected pixel.

A standard reference map pixel without a matching pixel in the algorithm skeleton was considered as a false-negative.

To evaluate the performance of the system, correct-detection rate, incorrect-detection rate, and missed-detection rate were computed. Correct-detection rate was defined as the number of correctly detected pixels over the number of standard reference map pixels. Missed-detection rate was defined as the number of false-negative pixels over the number of standard reference map pixels. Incorrectdetection rate was defined as the number of non-vessel-detected pixels over the number of pixels detected by the algorithm.

Those parameters were evaluated for the whole testing set and separately for images from data sets A and B. The Wilcoxon rank sum test was used to assess differences between the 2 subgroups. The test, equivalent to a Mann-Whitney $U$ test, performs a 2-sided rank sum test of the null hypothesis that data in the vectors $\mathbf{X}$ and $\mathbf{Y}$ are independent samples from identical continuous distributions 
TABLE 1. Vessel Detection Algorithm Performance

\begin{tabular}{lccc}
\hline & Correct-Detection Rate & Missed-Detection Rate & Incorrect-Detection Rate \\
\hline Overall & & & $12.8-40.5$ \\
$\quad$ Range & $78.0-99.3$ & $0.7-22.0$ & $26.9(8.0)$ \\
Mean (SD) & $88.6(6.0)$ & $11.4(6.0)$ & $27.1(0.11)$ \\
$\quad$ Median (IQR*) & $89.1(0.10)$ & $10.9(0.10)$ & \\
Data set A (14 fat-sat examinations) & & & \\
Range & $80.3-94.1$ & $5.9-19.7$ & $26.1-40.5$ \\
Mean (SD) & $88.3(4.4)$ & $11.7(4.4)$ & $28.0(8.5)$ \\
Median (IQR*) & $88.7(0.06)$ & $11.3(0.06)$ & \\
Data set B (14 non-fat-sat examinations) & & & \\
Range & $78.0-99.3$ & $0.7-22.0$ & $12.8-35.3$ \\
Mean (SD) & $88.9(7.5)$ & $11.1(7.5)$ & $24.9(7.0)$ \\
Median (IQR*) & $90.8(0.12)$ & $9.2(0.12)$ & $26.0(0.11)$ \\
\hline
\end{tabular}

*IQR indicates interquartile range.

with equal medians against the alternative hypothesis that they do not have equal medians. A $P \leq 0.05$ was considered statistically significant.

Moreover, the system's ability to reduce the number of vessel FPs of an automatic lesion detection method ${ }^{21}$ was also evaluated. To this purpose, the vessel mask generated by the algorithm was compared with the output of the CAD system. A CAD FP is defined as a connected region labeled as suspicious by the CAD but not confirmed by the radiologist, and a vessel FP indicates a FP corresponding to a vascular region. Every structure in the CAD output overlapping the algorithm's vessel mask by more than $90 \%$ was discarded. The reduction of the total number of vessel FPs of the CAD output was evaluated in percentage, and the Wilcoxon rank sum test was used to assess differences between the 2 subgroups.

\section{RESULTS}

\section{Vessel Detection Performance}

Algorithm performance was evaluated on a data set of 28 sequential DCE-MRI studies (testing set). The overall range, mean value, and median for correct-detection rate, missed-detection rate, and incorrect-detection rate are reported in Table 1. Thirteen patients had correct-detection rate greater than $90 \% ; 12$ patients, between $80 \%$ and $90 \%$; and 3 patients, less than $80 \%$. The incorrect-detection rate was less than $30 \%$ for 18 patients, between $30 \%$ and $40 \%$ for 9 patients, and greater than $40 \%$ for 1 patient. Incorrect detection was mainly caused by subtraction artifacts along the breast skin or the fibroglandular tissue. The missed-detection rate was less than $10 \%$ for 13 patients, between $10 \%$ and $20 \%$ for 12 patients, and greater than $20 \%$ for 2 patients. Missed detection was mainly caused by low-intensity vessels, especially near the end of the vascular tree. Figure 2 shows 2 examples of the algorithm results.

No significant differences were detected between the 2 subgroups $(P=0.50$ for correct-detection rate and missed-detection rate and $P=0.26$ for incorrect-detection rate).

\section{Reduction of FPs of a CAD System}

The total number of vessel FPs in the CAD output, the number of vessel FPs discarded by the vessel algorithm, and the percentage of reduction of vessel FPs of the CAD were calculated. Table 2 summarizes the results for the whole testing set. No statistical difference was found between the 2 subgroups $(P=0.50)$.

The sensitivity of the CAD system, defined as the number of lesions detected by the CAD system over the total number of lesions, was not affected by the vessel-detection algorithm because no lesions detected by the CAD were discarded after algorithm application.

\section{DISCUSSION}

Recently, an ipsilateral association between cancer and increased breast vascularity was demonstrated at contrast-enhanced breast MRI. Because breast carcinomas rely on the process of neoangiogenesis and the development of a complex network of blood microvessels for their growth, increased vascularity can be detected by MRI within the breast cancer lesion and the ipsilateral breast as a whole. Consequently, it has been postulated that scoring of breast vascularity could be used to increase specificity of breast MRI. ${ }^{12}$

The purpose of this study was to present a novel method for vessel extraction on breast DCE-MRI images, which can provide non-operator-dependent, reproducible vessel maps and reduce radiologists' reading time.

Several aspects of the presented method improved the 1 existing algorithm for blood vessel detection on breast DCE-MRI. ${ }^{8}$ Lin's method analyzed only 1 type of image, whereas the presented method achieves good results on a wider range of images for different types of lesions and acquisition parameters, such as the use of fatsaturation, and different contrast agents. In fact, the 2 breast MRI protocols used in this study can be considered quite representative of the spectrum of 1.5-T contrast-enhanced breast MRI protocols used in clinical practice because they consider 2 combinations of different magnetic resonance unit manufacturers, RF coils, types of RF sequences, and types of contrast materials. It seems reasonable that an algorithm for vessel extraction working for these 2 protocols should generally work for other protocols used for breast MRI in clinical practice as well. Lin's algorithm was tested on selected cases with large vessels from patients with relatively larger (mean [SD], 4.0 [2.1] cm), well-defined mass lesions, whereas the presented method also searches for relatively small vessels (diameters

TABLE 2. Reduction of Vessel FPs of the CAD system

\begin{tabular}{lccc}
\hline & $\begin{array}{c}\text { No. Vessel FP } \\
\text { of the CAD }\end{array}$ & $\begin{array}{c}\text { No. Vessel FP of the CAD } \\
\text { Discarded by the Algorithm }\end{array}$ & $\begin{array}{c}\text { \% of Vessel } \\
\text { FP Reduction }\end{array}$ \\
\hline Mean & 5.9 & 4.0 & 68.4 \\
SD & 4.0 & 2.8 & 30.0 \\
Median & 5.5 & 4.0 & 67.0 \\
\hline
\end{tabular}


between 1 and $2 \mathrm{~mm}$ ) on patients with smaller lesions [mean (SD), 2.4 $(2.0) \mathrm{cm}]$. This has, of course, an evident effect on the incorrectdetection rate (mean [SD] of $26.9 \%$ [8.0\%] for the proposed method and only a mean [SD] of $15.1 \%$ [7.8\%] for Lin's algorithm) because the search for small vessels entails the risk of including the fibroglandular tissue with similar enhancing values. The higher value of the incorrect-detection rate is, in any case, offset because the detection of vessels with a diameter between 1 and $2 \mathrm{~mm}$ is surely of much greater diagnostic interest than the detection of solely large vessels is.

Furthermore, Lin's algorithm relies on manual interaction to exclude the lesion, whereas the presented algorithm is fully automatic. Because lesions are not removed during vessel detection, some peripheral portion of the lesion may be incorrectly detected (Fig. 2B).

Finally, the algorithm was able to identify most vessels reported by the radiologist and improved the correct-detection rate by $10 \%$, with respect to Lin's study correct-detection rate.

Despite encouraging results, some limitations exist in the present study: $(a)$ the length of vessels, potentially useful to a more objective evaluation of breast vascularity, ${ }^{11}$ has not been considered; $(b)$ vessel branching points are often missed detected; $(c)$ the threshold set on the covariance eigenvalue ratio is empirically derived; $(d)$ subtraction artifacts along the breast boundary and skin are often incorrectly detected. Moreover, although the radiologist was highly experienced and the visual evaluation was semiquantitative, the substantial subjectivity of the reference standard should be considered.
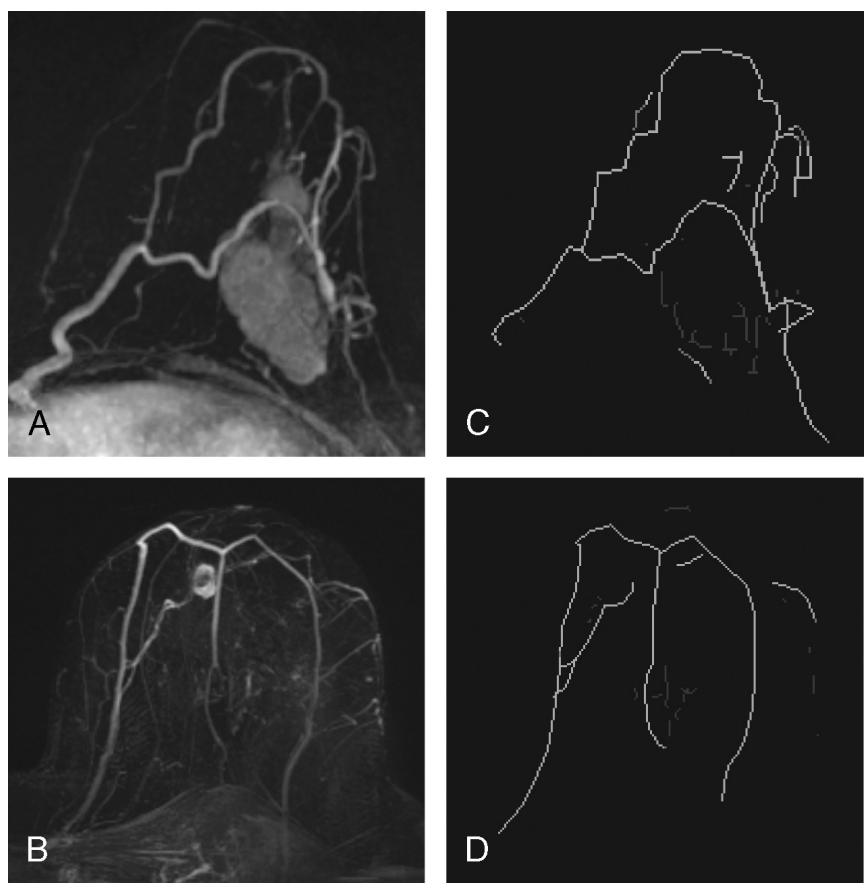

FIGURE 2. Examples of system performances for 1 study of group $A$ ( $A$ and $C$ ) and 1 study of group $B(B$ and $D)$. $A$ and $B$ are MIP images of the subtraction frame. $C$ and $D$ are the color validation maps obtained by comparison of the algorithm-detected vessels with the radiologist's vessel track. White pixels are correctly detected pixels, gray pixels are missed pixels and incorrectly detected pixels (non-vessel-detected pixels). For the study of images $A$ and $C$, correct-detection rate was $93.2 \%$, incorrect-detection rate was $16.9 \%$, and missed-detection rate was $6.8 \%$. For the study of images B and $\mathrm{D}$, correct-detection rate was $99.3 \%$, incorrect-detection rate was $18.5 \%$, and missed-detection rate was $7.0 \%$.
However, it might be very difficult to obtain an absolute criterion standard. In fact, not even morbid anatomy would help in this case because the volume of vessels of an excised breast could not be used as reference for an in-vivo 3-dimensional volumetric imaging modality.

The algorithm can be improved in several ways: $(a)$ by implementing a method based on connectivity to retrieve parts of vessels excluded because of low intensity, $(b)$ by adding a method to detect bifurcations and complex vessel structure based, for example, on both geometrical vesselness measure and vessel surface properties, ${ }^{22}(c)$ by studying a more robust threshold to be applied to the covariance eigenvalue ratio, and $(d)$ by including a method to recognize sheetlike structures, such as subtraction artifacts. Furthermore, to assess the clinical utility of this method, further validation is needed on a wider data set, including a larger lesion case study. Another issue to explore would be methods for extracting small vessels induced by tumor angiogenesis, which are often smaller than 1-mm diameter. To achieve this goal, both MRI protocol and algorithm parameters should be improved.

The reduction of vessel FPs of a CAD system was proposed as an example of how algorithm results are applied, but the vascular maps may be used for other purposes, such as for evaluation of the correlation of changes in vascularity with pathologic tumor response. A recent study, in fact, assessed the variation in whole-breast vascular maps on DCE-MRI after anthracycline- and taxane-based primary chemotherapy in patients with locally advanced breast cancer and encouraged the development of a standardized and highly reproducible method to extract and quantify breast vessels. ${ }^{23}$ Other 2 direct applications may be: $(a)$ to provide radiologists with automatic vascular maps, thus reducing the reading time and $(b)$ to reduce the labeling of vessels as suspicious regions in CAD systems, thus improving CAD specificity. In addition, it could also be useful for further vessel analysis on contrast-enhanced MRI images, such as the study of symmetry or asymmetry of vascularity or the study of adjacent lesions and feeding vessels.

In conclusion, a novel Hessian-matrix based method was proposed, which is able to extract blood vessels with a diameter greater than $1 \mathrm{~mm}$ on DCE-MRI images acquired with different sequences, acquisition parameters, and contrast agents. The reduction of vessel FPs of a CAD system was proposed as 1 application of the obtained results, but the algorithm vascular maps may find applications in other breast DCE-MRI vessel analysis that are waiting for a reliable quantification method.

\section{REFERENCES}

1. Mahoney MC, Bevers T, Linos E, et al. Opportunities and strategies for breast cancer prevention through risk reduction. CA Cancer J Clin. 2008;58:347-371.

2. Boyle P, Ferlay J. Cancer incidence and mortality in Europe, 2004. Ann Oncol. 2005;16:481-488

3. Ferlay J, Parkin DM, Steliarova-Foucher E. Estimates of cancer incidence and mortality in Europe in 2008. Eur J Cancer. 2010;46:765-781.

4. Sardanelli F, Giuseppetti GM, Canavese G, et al. Indications for breast magnetic resonance imaging: consensus document "Attualità in senologia," Florence 2007. Radiol Med. 2008;113:1085-1095.

5. Sardanelli F, Boetes C, Borisch B, et al. Magnetic resonance imaging of the breast: recommendations from the EUSOMA working group. Eur J Cancer. 2010;46:1296-1316.

6. Sardanelli F, Podo F, Santoro F, et al. Multicenter surveillance of women at high genetic breast cancer risk using mammography, ultrasonography, and contrastenhanced magnetic resonance imaging (the high breast cancer risk Italian 1 study): final results. Invest Radiol. 2011;46:94-105.

7. Fausto A, Magaldi A, Babaei Paskeh B, et al. MR imaging and proton spectroscopy of the breast: how to select the images useful to convey the diagnostic message. Radiol Med. 2007;112:1060-1068.

8. Lin M, Chen JH, Nie K, et al. Algorithm-based method for detection of blood vessels in breast MRI for development of computer-aided diagnosis. J Magn Reson Imaging. 2009;30:817-824.

9. Mahfouz AE, Sherif H, Saad A, et al. Gadolinium-enhanced MR angiography of the breast: is breast cancer associated with ipsilateral higher vascularity? Eur Radiol. 2001;11:965-969. 
10. Wright H, Listinsky J, Quinn C, et al. Increased ipsilateral whole breast vascularity as measured by contrast-enhanced magnetic resonance imaging in patients with breast cancer. Am J Surg. 2005;190:576-579.

11. Sardanelli F, Iozzelli A, Fausto A, et al. Gadobenate dimeglumine-enhanced MR imaging breast vascular maps: association between invasive cancer and ipsilateral increased vascularity. Radiology. 2005;235:791-797.

12. Schmitz AC, Peters NH, Veldhuis WB, et al. Contrast-enhanced 3.0-T breast MRI for characterization of breast lesions: increased specificity by using vascular maps. Eur Radiol. 2008;18:355-364.

13. Sardanelli F. Vessel analysis on contrast-enhanced MRI of the breast: global or local vascularity? AJR Am J Roentgenol. 2010;195:1246-1249.

14. Carriero A, Di Credico A, Mansour M, et al. Maximum intensity projection analysis in magnetic resonance of the breast. J Exp Clin Cancer Res. 2002; 21(suppl 3):77-81.

15. Fischer DR, Wurdinger S, Boettcher J, et al. Further signs in the evaluation of magnetic resonance mammography: a retrospective study. Invest Radiol. 2005; 40:430-435.

16. Dietzel M, Baltzer PA, Vag T, et al. The adjacent vessel sign on breast MRI: new data and a subgroup analysis for 1084 histologically verified cases. Korean $J$ Radiol. 2010;11:178-186.

17. Frangi AF, Niessen WJ, Vincken KL, et al. Multiscale vessel enhancement filtering. In: Wells WM, Colchester A and Delp SL, eds. Medical Image
Computing and Computer-Assisted Intervention-MICCAI'98, Lecture Notes in Computer Science. Vol 1496. Berlin, Germany: Springer Verlag; 1998: $130-137$.

18. Sato $\mathrm{Y}$, Nakajima S, Shiraga N, et al. Three-dimensional multiscale line filter for segmentation and visualization of curvilinear structures in medical images. Med Image Anal. 1998;2:143-168.

19. Gonzalez RC, Woods RE. Image Enhancement in the Spatial Domain. In: Gonzalez RC and Woods RE, eds. Digital image processing. 2nd ed. Upper Saddle River, NJ: Prentice Hall; 2002:125.

20. Nakayama R, Uchiyama Y, Yamamoto K, et al. Computer-aided diagnosis scheme using a filter bank for detection of microcalcification clusters in mammograms. IEEE Trans Biomed Eng. 2006;53:273-283.

21. Vignati A, Giannini V, De Luca M, et al. Performance of a fully automatic lesion detection system for breast DCE-MRI. J Magn Reson Imaging. 2011;34: 1341-1351.

22. Freiman M, Joskowicz L, Sosna J. A variational method for vessels segmentation: algorithm and application to liver vessels visualization. In: Proceedings of the Medical Imaging Conference of SPIE. Vol 7261. Orlando, FL: International Society for Optical Engineering; 2009:72610H-72618H.

23. Martincich L, Bertotto I, Montemurro F, et al. Variation of breast vascular maps on dynamic contrast-enhanced MRI after primary chemotherapy of locally advanced breast cancer. AJR Am J Roentgenol. 2011;196:1214-1218. 